\title{
Knuckle Cracking and Hand Osteoarthritis
}

\author{
Kevin deWeber, MD, FAAFP, Mariusz Olszewski, MD, and Rebecca Ortolano, MD
}

Background: Previous studies have not shown a correlation between knuckle cracking (KC) and hand osteoarthritis (OA). However, one study showed an inverse correlation between KC and metacarpophalangeal joint $\mathrm{OA}$.

Methods: We conducted a retrospective case-control study among persons aged 50 to 89 years who received a radiograph of the right hand during the last 5 years. Patients had radiographically proven hand $\mathrm{OA}$, and controls did not. Participants indicated frequency, duration, and details of their $\mathrm{KC}$ behavior and known risk factors for hand $\mathrm{OA}$.

Results: The prevalence of KC among 215 respondents (135 patients, 80 controls) was $20 \%$. When examined in aggregate, the prevalence of $\mathrm{OA}$ in any joint was similar among those who crack knuckles (18.1\%) and those who do not $(21.5 \% ; P=.548)$. When examined by joint type, $\mathrm{KC}$ was not a risk for $\mathrm{OA}$ in that joint. Total past duration (in years) and volume (daily frequency $\times$ years) of KC of each joint type also was not significantly correlated with $\mathrm{OA}$ at the respective joint.

Conclusions: A history of habitual KC-including the total duration and total cumulative exposuredoes not seem to be a risk factor for hand OA. (J Am Board Fam Med 2011;24:169-174.)

Keywords: Knuckle Cracking, Osteoarthritis

Knuckle cracking $(\mathrm{KC})$ is a behavior that involves manipulation of the finger joints that results in an audible crack, and it is often done habitually. Prevalence estimates vary between $25 \%$ and $54 \%$, depending on the population studied. ${ }^{1,2}$ The behavior can become habitual because of immediate joint tension release and increased joint range of motion. $^{3}$

During an attempt to crack a knuckle, the joint is manipulated by axial distraction, hyperflexion, hyperextension, or lateral deviation. This lengthens part or all of the joint space and greatly decreases

This article was externally peer reviewed.

Submitted 6 July 2010; revised 15 October 2010; accepted 22 October 2010.

From the Department of Family Medicine, Uniformed Services University of the Health Sciences, Bethesda, MD (KD); the Department of Radiology, National Naval Medical Center, Bethesda, MD (MO); and the Madigan Army Medical Center, Tacoma, WA (RO).

Funding: Uniformed Services University of the Health Sciences Grant HU81CV.

Conflict of interest: none declared.

Disclaimer: The opinions contained herein are those of the authors. They do not reflect official policy of the Department of Defense, the Department of the Navy, or the Uniformed Services University.

Corresponding author: Kevin deWeber, MD, FAAFP, USUHS, Dept. FAP, 4301 Jones Bridge Road, Bethesda, MD 20814 (E-mail: kdeweber@usuhs.mil). intra-articular pressure, causing gases that have dissolved in the synovial fluid to form microscopic bubbles, which coalesce. When the joint space reaches its maximum distraction (up to 3 times its resting joint space distance), joint fluid rushes into the areas of negative pressure. The larger bubbles suddenly collapse into numerous microscopic bubbles, leading to the characteristic cracking sound. The maneuver leaves the joint space wider than it had been and synovial fluid more widely distributed. The stretching of joint ligaments required to produce the widened joint space also leaves the joint with greater range of motion. It typically takes at least 15 minutes for the joint to be able to be cracked again because of the time required for the microscopic bubbles to fully dissolve into solution and for the joint space to retract back to its resting position. $^{4}$

Common urban legend suggests that $\mathrm{KC}$ will lead to arthritis of the hand joints. Adverse effects of $\mathrm{KC}$ have been cited but are not well supported in the medical literature. Case reports of acute joint damage from unusually vigorous and deviant $\mathrm{KC}$ attempts are rare. One reported a thumb ulnar collateral ligament sprain and a fifth finger extensor tendon sagittal band tear at the metacarpophalan- 
geal (MCP) joint. ${ }^{5}$ Another described a man with chondrocalcinosis in the first and fourth MCP joints and ligamentous ossification in the third MCP joint, presumably caused by chronic KC. ${ }^{6}$

The amount of force required to crack a knuckle has been shown in vitro studies to exceed the energy threshold that can lead to articular cartilage damage. $^{7}$ The cavitation effect of intra-articular bubble formation and collapse is also mechanically similar to cavitation of ship propellers, a process that has been shown to produce wear on the propeller surfaces. ${ }^{1}$ Based on these facts, it is logical to theorize that habitual $\mathrm{KC}$ may lead to gradual thinning of articular cartilage and eventual clinical osteoarthritis (OA). However, this claim remains unsubstantiated in the medical literature.

A MEDLINE search using keywords "joint cracking" and "knuckle cracking" revealed 2 studies that addressed the incidence of $\mathrm{OA}$ in knuckle crackers. The first looked at 300 randomly selected persons older than age 45 (mean age, 63 years). Participants were assessed by a questionnaire and a physical examination of the hands. Those who cracked knuckles were more likely to have hand swelling and reduced grip strength, but the prevalence of hand $\mathrm{OA}$ was $16 \%$ among those who cracked knuckles and those who did not. ${ }^{2}$ The investigators did not specify which joints were cracked nor which joints were affected with OA.

Another study involved examination of the hand radiographs of 28 nursing home residents (average age, 78 years). Participants were asked to recall if they currently or previously cracked knuckles, but investigators did not specify which joints. In this study, KC of the MCP joint was found to be negatively correlated with $\mathrm{OA}$. The prevalence of $\mathrm{KC}$ in the 6 persons with OA of the MCP joint was $17 \%$, whereas the prevalence of $\mathrm{KC}$ in the group without OA of the MCP joint was $64 \%$. This suggests that KC may be associated with a lower prevalence of OA at the MCP joint. ${ }^{1}$

Though somewhat useful, neither of these studies specified which joints participants cracked. Neither quantified the duration or frequency of $\mathrm{KC}$, both of which could have correlations with the presence or absence of OA. Duration and frequency may be relevant because, based on the mechanical logic above, the more times that the maneuver is performed, the more the risk of mechanical wear on affected surfaces would, theoretically, increase.
$\mathrm{OA}$ of the hand increases in prevalence and severity with age. ${ }^{8}$ The prevalence of symptomatic hand OA has been reported to be $22 \%$ in persons age 71 to 100 years among the general population. ${ }^{9}$ Other risk factors include prior joint trauma, family history of hand OA, and history of heavy labor involving the hands. ${ }^{10}$ Those with hand OA have reduced maximal grip strength, more difficulty writing and handling small objects, and more difficulty carrying objects. ${ }^{8}$ Given this burden of suffering from hand $\mathrm{OA}$ and the lack of curative or disease-modifying treatments, factors that potentially protect against $\mathrm{OA}$ warrant further investigation. One such factor is knuckle cracking.

\section{Materials and Methods}

After approval by the Uniformed Services University of the Health Sciences and the National Naval Medical Center institutional review boards in Bethesda, MD, we conducted a retrospective, nested, case-control study to examine $\mathrm{KC}$ behavior in a population aged 50 to 89 years. Participants were selected from patients who had received radiographs of the right hand within the 5-year period from October 2003 to October 2008. Patients consisted of persons with hand $\mathrm{OA}$; the controls did not have OA, based on radiographic diagnosis. Gathering data about only one hand is easier and still allows accurate assessment of KC prevalence. Using power analysis we determined that we would need approximately 200 participants to reach statistical significance.

We identified eligible patients with hand OA by querying the military electronic medical record database for persons with International Classification of Diseases 9 codes of 715.04 (OA of hand generalized), 715.14 (OA of hand localized primary), 715.34 (OA of hand localized), and 715.94 (OA of hand). We reviewed reports of their hand radiographs to ensure that hand $\mathrm{OA}$ was present and to determine which joints were affected-the distal interphalangeal (DIP), proximal interphalangeal (PIP), MCP, and/or the first carpometacarpal (1CMC) joints. We then searched the same population in the database for persons who did not have International Classification of Diseases 9 diagnoses of hand OA. Those whose reports of their hand radiographs confirmed the absence of OA or the presence of 
any exclusion criteria were selected as controls. We excluded persons who did not have a written report of a right-hand radiograph within the specified 5-year period and those with diagnoses of inflammatory arthritidies. We identified 141 controls without hand OA and 188 patients with OA, for a total of 329 participants. The participant selection process is summarized in Figure 1.

Each participant was assigned a unique study number and was mailed a packet containing the study questionnaire. Participants were asked to recall and specify which joints on their right hand they crack, using a drawing of a hand and indicating locations of joints to assist them. We categorized these into DIP, PIP, MCP, and 1CMC joint types. In addition to $\mathrm{KC}$ duration, we asked participants to quantify their frequency of $\mathrm{KC}$ daily, divided into 5 ordinal categories (none, 1-5 times/day, 6-10 times/day, 10-20 times/day, and $>20$ times/ day). Participants were also asked about specific risk factors for OA, including family history of hand OA, any fractures or dislocations of particular joints, and a history of heavy labor using the hands for $>5$ years. Persons with OA were asked about the presence and duration of their hand OA symptoms.

We used basic descriptive statistics to characterize respondent demographics. To investigate any association between $\mathrm{KC}$ and $\mathrm{OA}$, we per-

Figure 1. Selection of participants. OA, osteoarthritis.

\section{OA CASES}

Database search: 2,330 records, age 50-89, $x$-rays within 5 years, ICD-9 diagnoses of OA
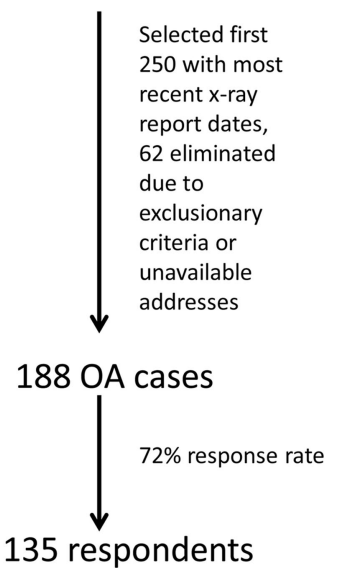

\section{CONTROLS}

Database search: 859 records, age 50-89, $\mathrm{x}$-rays within 5 years, no ICD-9 diagnoses OA

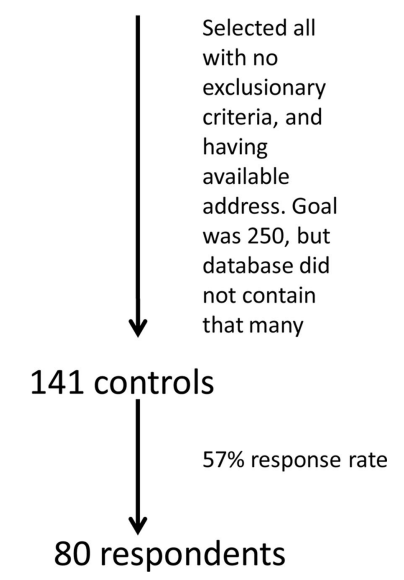

Table 1. Presence of Other-Hand Osteoarthritis (OA) Risk Factors among Patients and Controls

\begin{tabular}{lccr}
\hline & $\begin{array}{c}\text { Patients with } \\
\text { Hand OA } \\
(\mathrm{n})\end{array}$ & $\begin{array}{c}\text { Controls } \\
(\mathrm{n})\end{array}$ & $P$ \\
\hline Risk Factor & $64.4(135)$ & $57.0(80)$ & $<.001$ \\
Age (years) & $74.8(101)$ & $68.8(55)$ & .335 \\
$\begin{array}{l}\text { Female sex (\%) } \\
\text { Family history } \\
\text { of hand OA }\end{array}$ & $48.9(66)$ & $32.5(26)$ & .039 \\
$>5$ years heavy & $19.3(26)$ & $16.2(13)$ & .857 \\
labor & & & \\
\hline
\end{tabular}

Values presented as \% (n).

formed Pearson $\chi^{2}$ tests between the prevalence of $\mathrm{KC}$ in controls and participants with OA. To explore the relationship of $\mathrm{OA}$ with the duration (in years) of $\mathrm{KC}$ for each joint type, we used independent sample $t$ tests to compare the mean duration of $\mathrm{KC}$ in controls and those with $\mathrm{OA}$.

\section{Results}

We received 215 surveys from the 329 participants (135 from patients with $\mathrm{OA}$ and 80 from controls), for a response rate of $65 \%$. The mean age of respondents was 62 years (SD, 8 years). Of the 215 respondents, 43 indicated they crack their knuckles (20\% prevalence). Women were less likely to habitually crack knuckles than men: $17 \%(\mathrm{n}=26)$ versus $29 \%(\mathrm{n}=17)$, respectively $(P=.05)$. The presence of other risk factors for hand OA among patients and controls is summarized in Table 1 .

The most commonly cracked joint was the PIP $(15.9 \% ; \mathrm{n}=34)$, followed by the MCP $(13.5 \%$; $\mathrm{n}=29)$, DIP $(6.1 \% ; \mathrm{n}=13)$, and $1 \mathrm{CMC}(2.3 \%$; $\mathrm{n}=5$ ) joints. Based on analysis of the radiograph reports of all participants, we also determined which joint types were affected with OA. When participants indicated a history of trauma to a specific joint, we excluded OA data only for that joint. The joint most commonly affected by OA was the DIP joint $(68.4 \% ; \mathrm{n}=91)$, followed by the $1 \mathrm{CMC}(57.1 \% ; \mathrm{n}=76), \operatorname{PIP}(54.1 \% ; \mathrm{n}=$ $72)$, and MCP (28.6\%; $\mathrm{n}=38)$ joints.

Table 2 lists the prevalence and duration of $\mathrm{KC}$ at each specific joint type among the $\mathrm{OA}$ versus control groups. The prevalence of any $\mathrm{KC}$ among the controls $(23.2 \% ; \mathrm{n}=19)$ was not significantly different from prevalence in persons with $\mathrm{OA}(18.0 \% ; \mathrm{n}=24 ; P=.361)$. When 
Table 2. Prevalence and Duration of Habitual Knuckle Cracking in Patients and Controls by Joint Type

\begin{tabular}{|c|c|c|c|}
\hline Joint Cracked & Controls & Patients with $\mathrm{OA}$ & $P$ \\
\hline DIP (\% [n]) & $9.2(11)$ & $2.2(2)$ & .097 \\
\hline $\begin{array}{l}\text { Duration* } \\
\text { (years) }\end{array}$ & 26.3 & 36.0 & .257 \\
\hline PIP (\% [n]) & $16.1(23)$ & $15.5(11)$ & .911 \\
\hline $\begin{array}{c}\text { Duration* } \\
\text { (years) }\end{array}$ & 28.3 & 21.6 & .556 \\
\hline $\operatorname{MCP}(\%[\mathrm{n}])$ & $14.3(25)$ & $10.0(4)$ & .474 \\
\hline $\begin{array}{c}\text { Duration* } \\
\text { (years) }\end{array}$ & 26.4 & 25.0 & .764 \\
\hline $1 \mathrm{CMC}(\%[\mathrm{n}])$ & $2.9(4)$ & $1.3(1)$ & .746 \\
\hline $\begin{array}{l}\text { Duration* } \\
\text { (years) }\end{array}$ & Data unavailable & Data unavailable & \\
\hline Any joint & $23.2(19)$ & $18.0(24)$ & .361 \\
\hline
\end{tabular}

*Independent sample $t$ test was used.

OA, osteoarthritis; DIP, distal interphalangeal; PIP, proximal interphalangeal; MCP, metacarpophalangeal; $1 \mathrm{CMC}$, first carpometacarpal.

examined by specific joint type, prevalence of $\mathrm{KC}$ in the DIP, PIP, MCP, and 1CMC joints was similar in patients and controls. For the DIP, PIP, and MCP joints, there were no significant associations between duration of $\mathrm{KC}$ and the presence of OA. There was insufficient data for the $1 \mathrm{CMC}$ joint.

We next explored the relationship of OA with the total volume of $\mathrm{KC}$ behavior to which each joint type was exposed. For each frequency category we interpolated to facilitate calculations. The category " 1 to 5 times/day" was scored as 3 ; "6 to 10 times/day" was scored as 8 ; and " 10 to 20 times/day" was scored as 15 . No participants cracked knuckles more frequently than this. The product of frequency $\times$ duration (years) resulted in an estimation of total $\mathrm{KC}$ exposure-what we call "crack-years," similar to the quantification of tobacco use in pack-years. We calculated this for each joint type.

The mean total KC exposure for the DIP joint was 108 crack-years (SD, 17 crack-years). An independent sample $t$ test failed to reveal a significant association between DIP crack-years and the presence or absence of OA $(P=.418)$. The mean KC exposure in the PIP joint was 70 crackyears (SD, 44 crack-years) and, again, there was no significant association between crack-years of the PIP joint among the OA group compared with the control group $(P=.214)$. The mean MCP joint exposure was 75 crack-years (SD, 52 crack-years), with no significant relationship to OA $(P=.503)$. There were insufficient data for the 1CMC joint.

We attempted to determine "which came first: the OA or the $\mathrm{KC}$ " by asking patients with $\mathrm{OA}$ to indicate how many years have they noticed OA symptoms. Many patients with OA were asymptomatic or did not answer. However, for those who did answer and who also cracked knuckles, we used the paired-samples $t$ test to compare the duration of $\mathrm{KC}$ to the duration of $\mathrm{OA}$ symptoms and found that, for all joint types, the duration of $\mathrm{KC}$ was significantly greater than the duration of OA symptoms (see Table 3 ).

Lastly, we performed binary logistic regression analyses to evaluate the relative contributions of $\mathrm{KC}$, age, sex, family history of hand OA, and history of prolonged heavy labor to the risk of hand OA. Analyses confirmed a strong association between family history of $\mathrm{OA}$ and the presence of hand OA (odds ratio, 2.98; $P=.009$ ) and between age and hand $\mathrm{OA}$ (odds ratio, 1.20 per additional year of age; $P<.001)$. Knuckle cracking, sex, and heavy labor were not associated with $\mathrm{OA}(P=.626$, $P=.222$, and $P=.632$, respectively) after controlling for other risk factors.

\section{Discussion}

This study represents the most comprehensive evaluation to date of habitual KC and any association with hand OA. Our findings support the conclusions of 2 previous studies ${ }^{1,2}$ that the presence of $\mathrm{KC}$ is not associated with hand OA. Ours is the first study to correlate the duration and the total volume of previous $\mathrm{KC}$ with $\mathrm{OA}$, in addition to the presence or absence of KC. Participants

Table 3. Comparison of Habitual Knuckle Cracking Duration (Any Frequency) with Duration of Hand Osteoarthritis (OA) Symptoms Using Paired Samples $t$ Test

\begin{tabular}{lccc}
\hline Joint (n) & $\begin{array}{c}\text { Duration of OA } \\
\text { Symptoms (Years) }\end{array}$ & $\begin{array}{c}\text { Duration of KC } \\
\text { (Years) }\end{array}$ & $P$ \\
\hline DIP (7) & 7.6 & 27.1 & .006 \\
PIP (19) & 9.7 & 25.1 & .001 \\
MCP (15) & 7.9 & 23.1 & .003 \\
1CMC (3) & 8.0 & 33.3 & .091 \\
\hline
\end{tabular}

OA, osteoarthritis; DIP, distal interphalangeal; PIP, proximal interphalangeal; MCP, metacarpophalangeal; 1CMC, first carpometacarpal; KC, knuckle cracking. 
described how frequently each day they crack each type of knuckle and for how many years they have been doing it. First, our results indicated that the duration of $\mathrm{KC}$ has no correlation to the presence of OA in the DIP, PIP, and MCP joints. We also calculated "crack-years," which roughly quantified the total amount of exposure to this behavior. This allowed investigation of a possible "dose-response" relationship between the mechanical effects of $\mathrm{KC}$ and OA. Again, when looking at $\mathrm{KC}$ of each joint type, we found no significant correlation of KC "crack-years" with $\mathrm{OA}$ in the respective joint.

Our study methodology, though useful to find large enough numbers of participants with and without $\mathrm{OA}$ to generate adequate statistical power, does have limitations. Our sample was limited to people who presented to a health care facility-likely with hand symptoms-and who received a radiograph of their hand. Radiographic diagnosis of OA is preferred over physical examination because radiograph findings often will appear before physical examination findings. The time frame of 5 years was somewhat arbitrary but reflected a period of time in which only minor radiographic changes to $\mathrm{OA}$ in the hand joints might occur. We chose the age range of 50 to 89 years to find enough patients with OA and enough people who had been cracking knuckles for many years. Limiting analysis to only the right hand was also somewhat arbitrary but was scientifically sound because knuckle cracking is typically a bilateral behavior.

The use of participants who received radiographs of their hand during the last 5 years introduced a selection bias that affects the generalizability of our results. This also likely explains the lower prevalence of $\mathrm{KC}$ in our study population $(20 \%)$ compared with other studies of less selective populations. Prevalence reported in other studies included $25 \%$ in adults older than 45 years, ${ }^{5} 34 \%$ in 11-year-old children, ${ }^{1}$ and $54 \%$ among nursing home residents with a mean age of 78 years. ${ }^{1}$ The subset of persons in our study does not accurately reflect the general population, most of which do not have hand symptoms. However, because both cohorts in this study met the same inclusion and exclusion criteria, biases were likely similar in both and probably will not diminish the usefulness of differences between the 2 cohorts. Future studies that randomly select asymptomatic persons for ra- diographs of the hand would more accurately reflect the general population, though this methodology would subject large numbers of people to the risks of radiation.

What we do not know yet are all the reasons why people crack their knuckles and the effect this has on their joints in the long term. Though some people may start KC because of joint symptoms, patients with $\mathrm{OA}$ in our study started $\mathrm{KC}$ long before the onset of OA symptoms. Some people may crack knuckles because of the sense of relief it can bring, some because of habit, and some from both. People may stop KC when hand symptoms appear either because of fear of what KC might do to their joints or because $\mathrm{KC}$ becomes too uncomfortable. These are all factors that were not accounted for in this study but contribute to the complex nature of this behavior. We did not ascertain if $\mathrm{KC}$ was painful, neutral, or even relieved OA symptoms, so we do not know the reason for the difference.

What we can conclude, however, is that, in these cohorts of persons aged 50 to 89 years, a history of habitual KC-including the total duration and total cumulative exposure to $\mathrm{KC}$ - does not seem to be a risk factor for hand OA.

The authors would like to thank Cara Olsen, Assistant Professor of Preventive Medicine and Biometrics at Uniformed Services University; and Roberta Williams, National Capital Area IT Management Specialist, for their invaluable assistance with data management.

\section{References}

1. Swezey RL, Swezey SE. The consequences of habitual knuckle cracking. West J Med 1975;122:377-9.

2. Castellanos J, Axelrod D. Effect of habitual knuckle cracking on hand function. Ann Rheum Dis 1990; 49:308-9.

3. Protopapas MG, Cymet TC. Joint cracking and popping: understanding noises that accompany articular release. J Am Osteopath Assoc 2002;102: 283-7.

4. Unsworth A, Dowson D, Wright V. 'Cracking joints.' A bioengineering study of cavitation in the metacarpophalangeal joint. Ann Rheum Dis 1971; 30:348-58.

5. Chan PS, Steinberg DR, Bozentka DJ. Consequences of knuckle cracking: a report of two acute injuries. Am J Orthop 1999;28:113-4.

6. Watson P, Hamilton A, Mollan R. Habitual joint cracking and radiological damage. BMJ 1989;299: 1566. 
7. Watson P, Kernohan WG, Mollan RA. A study of the cracking sounds from the metacarpophalangeal joint. Proc Inst Mech Eng 1989;203:109-18.

8. Kallman DA, Wigley FM, Scott WW Jr, Hochberg MC, Tobin JD. The longitudinal course of hand osteoarthritis in a male population. Arthritis Rheum 1990;33:1323-32.
9. Zhang Y, Niu J, Kelly-Hayes M, Chaisson CE, Aliabadi P, Felson DT. Prevalence of symptomatic hand osteoarthritis and its impact on functional status among the elderly. Am J Epidem 2002;156:1021-7.

10. Wilder FV, Barrett JP, Farina EJ. Joint-specific prevalence of osteoarthritis of the hand. Osteoarthritis Cartilage 2006;14:953-7. 